\title{
Direct clipping of large basilar trunk aneurysm
}

Toshikazu Kimura, M.D., Daichi Nakagawa, M.D., ${ }^{2}$ and Kensuke Kawai, M.D., Ph.D. ${ }^{2}$

1Department of Neurosurgery, NTT Medical Center Tokyo; and 2Department of Neurosurgery, the University of Tokyo Hospital, Tokyo, Japan

A large basilar trunk aneurysm was incidentally found in a 77-year-old woman in examination for headache. Though it was asymptomatic, high signal intensity was noticed in the brainstem around the aneurysm on FLAIR image of MRI. As she was otherwise healthy, surgical clipping was performed through anterior temporal approach.

The video can be found here: http://youtu.be/0soWM8meCW8.

KEY WORDS clipping; basilar trunk aneurysm; 3D simulation 